九州大学学術情報リポジトリ

Kyushu University Institutional Repository

\title{
RENEWAL THEORETICAL APPROACH TO THE MISSION RELIABILITY OF A REDUNDANT REPAIRABLE SYSTEM WITH TWO DISSIMILAR UNITS
}

Kodama, Masanor i

Sheffield University | Osaka University

Fukuta, Jiro

Gifu University

https://doi.org/10.5109/13098

出版情報: 統計数理研究. 16 (3/4)，pp.103-114，1975-03. Research Association of Statistical Sciences

バージョン :

権利関係 : 


\title{
RENEWAL THEORETICAL APPROACH TO THE MISSION RELIABILITY OF A REDUNDANT REPAIRABLE SYSTEM WITH TWO DISSIMILAR UNITS*
}

\author{
By \\ Masanori Kodama** and Jiro FuKUTA***
}

(Received October 30, 1974)

\begin{abstract}
A system consisting of two dissimilar, redundant, repairable units is considered. We shall say that a major breakdown occurs in the system when both units fails. The system fails if one unit under repair is not repaired within a fixed time measured from the instant at which major breakdown occurs, or if the number of major breakdowns during the mission period exceeds a fixed number. As a special case, this number is allowed to be "infinite".

The Laplace transforms of the reliability and the mean time to system failure are derived, and the explicit formulas in the special cases are exhibited.
\end{abstract}

\section{§1. Model definition.}

1. The system consists of two dissimilar redundant units $A_{1}$ and $A_{2}$.

2. There is only one repair station. When one unit fails, its repair begins at once, and when two units fail simultaneously, unit $A_{i}$ is sent for repair with a specified constant probability $\alpha_{i}, i=1,2$, where $\alpha_{1}+\alpha_{2}=1$.

Concerning failure and repair we assume the following:

3. When the two units are good, unit-failures occur as three independent Poission processes with failure rates $\lambda_{1}, \lambda_{2}$ and $\lambda_{12}$. Events in the process with rate $\lambda_{i}$ cause failure of unit $A_{i}$ only, and events in the process with rate $\lambda_{12}$ cause simultaneous failure of unit $A_{1}$ and $A_{2}$.

4. When only one unit, $A_{i}$ is good, failure of the unit is Poisson with rate $\lambda_{\imath}^{\prime}, \lambda_{i}^{\prime} \neq \lambda_{i}$.

5. The repair time for each unit, $A_{i}$ is independently distributed with general probability density function $f_{i}(t)$, but must be well behaved enough for the appropriate analytic operations to be performed.

6. The failure and repair processes for the two units are entirely independent.

7. The repaired unit is considered to be new again.

* This research was supported by the Science Research Council under Grant No. 4349/00.

** Sheffield University, Sheffield and Osaka University, Osaka.

*** Gifu University, Gifu. 
8. A major breakdown occurs when both units fail. And the system fails if one unit under repair is not repaired within a fixed time, $\tau$, measured from the instant at which major breakdown occurs. The number of major breakdowns during the mission period is limited as follows: We assume that for fixed $n$, any unit under repair after the $(n+1)$-st major breakdown is not repaired within allowable down time with probability 1 . Therefore the system also fails if the number of major breakdown in $T-\tau$ exceeds $n$.

Recently J. Fukuta and M. Kodama [1] gave a mission reliability for a redundant repairable system with two dissimilar units using the method of supplementary variables. In [1], we assumed that the number of failures during the mission period is "infinite". In this paper, we assume that the number of major breakdowns during the mission period is a fixed number and is allowed to be "infinite". We study the above mentioned model by renewal theoretical approach introducing the probability functions depending on the number of major breakdowns. Our results include the results in [1] as special cases.

\section{$\S 2$. Equations of the system.}

\section{Notation List :}

$i \quad$ subscript describing the 2 which $i=1,2$

$\alpha_{i} \quad$ probability that unit $A_{i}$ is sent for repair, when both units fail simultaneously (see $2, \S 1$ )

$\lambda_{i}, \lambda_{i}^{\prime}, \lambda_{12}$ failure rates (see $\left.3, \S 1\right)\left(\lambda_{i}>0, \lambda_{i}^{\prime}>0, \lambda_{12} \geqq 0\right)$

$\tau \quad$ allowed down time (see $\S 1$ )

$n \quad$ maximum number of major breakdown permitted during the mission period

$f_{i}(t) \quad$ pdf of repair time of unit $A_{i}$

$F_{i}(t) \quad$ cdf of repair time of unit $A_{i}$

$f^{*}(s) \quad$ Laplace transform of $f(t)$

$f^{\dagger}(s) \quad$ Integral $\int_{0}^{\tau} e^{-s t} f(t) d t$

$\delta_{i j} \quad$ Kronecker symbol

$\alpha_{i}^{*}(s) \quad=s+\lambda_{1}+\lambda_{2}+\lambda_{12}-\lambda_{i} f_{i}^{*}\left(s+\lambda_{3-i}^{\prime}\right)$

$\beta_{i}^{*}(s ; \tau)=\left[e^{\lambda_{3-1}^{\prime} \tau}-1\right] f_{i}^{*}\left(s+\lambda_{3-i}^{\prime}\right)-e^{\lambda_{3-i}^{\prime}} f_{i}^{\dagger}\left(s+\lambda_{3-i}^{\prime}\right)+f_{i}^{\dagger}(s)$

$\delta_{i}^{*}(s ; \tau)=\lambda_{3-i}^{\prime} e^{-s \tau}\left\{e^{\left(s+\lambda_{3-i}^{\prime}\right)}\left[f_{i}^{*}\left(s+\lambda_{3-i}^{\prime}\right)-f_{i}^{\dagger}\left(s+\lambda_{3-i}^{\prime}\right)\right]-f_{i}^{*}\left(s+\lambda_{3-i}^{\prime}\right)\right.$ $\left.+F_{i}(\tau)\right\} /\left[s\left(s+\lambda_{3-i}^{\prime}\right)\right]-\beta_{i}^{*}(s ; \tau) / s$

$\gamma_{i}^{*}(s ; \tau)=\left[s+\lambda_{3-i}^{\prime}-\lambda_{3-i} e^{-s \tau}\right]\left[1-f_{i}^{*}\left(s+\lambda_{3-i}^{\prime}\right)\right] /\left[s\left(s+\lambda_{3-i}^{\prime}\right)\right]$ $+f_{i}^{*}\left(s+\lambda_{3-i}^{\prime}\right)\left[s+\lambda_{12}\left(1-e^{-s \tau}\right)\right] /\left[s\left(s+\lambda_{1}+\lambda_{2}+\lambda_{12}\right]\right.$

$\varepsilon_{i}^{*}(s ; \tau)=\left[1-\beta_{i}^{*}(s ; \tau)-f_{i}^{*}\left(s+\lambda_{3-i}^{\prime}\right)\right] /\left(s+\lambda_{3-i}^{\prime}\right)+\lambda_{3-i}^{\prime}\left[1-f_{i}^{\dagger}(s)-e^{-s \tau}\left(1-F_{i}(\tau)\right)\right] /\left[s\left(s+\lambda_{3-i}^{\prime}\right)\right]$ $k_{i}^{*}(s ; \tau)=\lambda_{3-i}+\lambda_{12} \alpha_{i} f_{i}^{\dagger}(s)$

$\eta^{*}(s ; \tau)=\left\{s+\lambda_{12} \sum_{j=1}^{2} \alpha_{j}\left[1-f_{j}^{\dagger}(s)-e^{-s z}\left(1-F_{j}(\tau)\right)\right]\right\} / s$

$E_{2} \quad$ denotes unit $A_{i}$ and unit $A_{3-i}$ in operation

$E_{1}(i)$ denotes unit $A_{3-i}$ in operation and unit $A_{i}$ under repair

$E_{0}(i) \quad$ denotes unit $A_{i}$ under repair and unit $A_{3-i}$ queneing for repair 
$q_{2}^{j}(u) \quad$ probability that system is state $E_{2}$ at $u=0$, has exactly $j$ major breakdowns during the interval 0 to $u$, and no major breakdowns has lasted longer than $\tau$. The major breakdown if it occured in time interval $(T-\tau, T)$ is not included (the failures during this interval do not count)

$q_{k(i)}^{j}(u) \quad$ denotes probability similar to the $q_{2}^{j}(u)$, except the system has just entered the state $E_{k}(i)(k=0,1)$ at $u=0$ and when $k=0$, the initial failed state is not included in $j$.

$Q_{2}^{n}(u) \quad$ sum of $q_{2}^{j}(u)$ from $j=0$ to $j=n$

$Q_{k(i)}^{n}(u) \quad$ sum of $q_{k(i)}^{j}(u)$ from $j=0$ to $j=n$

$\mathrm{MTSF}_{2}^{n}, \mathrm{MTSF}_{k(i)}^{n}$ notations are analogous to $Q_{2}^{n}(u)$ and $Q_{k(i)}^{n}$ respectively. They are easy to show that $\operatorname{MTSF}_{2}^{n}=\left.Q_{2}^{* n}(s)\right|_{s=0}, \operatorname{MTSF}_{k(i)}^{n}=\left.Q_{k(i)}^{* n}(s)\right|_{s=0}$.

For $u<\tau, q_{2}^{0}(u)=1, q_{3}^{j}(u)=0(j \geqq 1) ; q_{k(i)}^{0}(u)=1, q_{k(i)}^{j}(u)=0(k=0,1 ; i=1,2 ; j \geqq 1)$, and by definition of $q_{2}^{j}(u)$ and $q_{k(i)}^{j}(u)$, the following set of integral equations of this system can be easily set up:

$$
\begin{aligned}
& q_{2}^{0}(T)=e^{-\left(\lambda_{1}+\lambda_{2}+\lambda_{12}\right)(T-\tau)} \\
& +\int_{0}^{T-\tau} e^{-\left(\lambda_{1}+\lambda_{2}+\lambda_{12}\right) x}\left[\lambda_{1} q_{1(1)}^{0}(T-x)+\lambda_{2} q_{1(2)}^{0}(T-x)\right] d x, \quad T \geqq \tau, \\
& =1 \text {, } \\
& q_{1(i)}^{0}(T)=e^{-\lambda_{3-i}^{\prime}(T-\tau)}\left[1-F_{i}(T-\tau)\right]+\int_{0}^{T-\tau} e^{-\lambda_{3-i}^{\prime} x} f_{i}(x) q_{2}^{0}(T-x) d x, \quad T \geqq \tau, \\
& =1 \text {, } \\
& q_{0(i)}^{0}(T)=\int_{0}^{\bar{\tau}} f_{i}(x) q_{1(3-i)}^{0}(T-x) d x \\
& =1 \text {, } \\
& q_{2}^{j}(T)=\int_{0}^{T-\tau} e^{-\left(\lambda_{1}+\lambda_{2}+\lambda_{12}\right) x}\left\{\lambda_{1} q_{1(1)}^{j}(T-x)+\lambda_{2} q_{1(2)}^{j}(T-x)\right. \\
& \left.+\lambda_{12}\left[\alpha_{1} q_{0(1)}^{j-1}(T-x)+\alpha_{2} q_{0(2)}^{j-1}(T-x)\right]\right\} d x, \quad T \geqq \tau, \\
& =0 \text {, } \\
& q_{1(i)}^{j}(T)=\int_{0}^{T-\tau} \int_{y=0}^{\tau} \lambda_{i-i}^{\prime} e^{-\lambda_{i-i}^{\prime} x} f_{i}(x+y) q_{1(3-i)}^{j-1}(T-x-y) d y d x \\
& +\int_{0}^{T-\tau} e^{-\lambda_{3-i}^{\prime} x} f_{i}(x) q_{2}^{j}(T-x) d x, \quad T \geqq \tau, \\
& =0 \text {, } \\
& q_{0(i)}^{j}(T)=\int_{0}^{\tau} f_{i}(x) q_{1(3-i)}^{j}(T-x) d x, \\
& \text { otherwise . } \\
& T \geqq \tau, \\
& =0 \text {, }
\end{aligned}
$$

\section{§ 3. Solution of the problem.}

We solve this set of equations by employing the Laplace transform technique. Taking Laplace transforms of both of $(1)-(6)$, we have ${ }^{(1 *)}$ 


$$
\begin{aligned}
& q_{2}^{* 0}(s)=\left\{s+\lambda_{12}\left(1-e^{-s \tau}\right)+s\left[\lambda_{1} q_{1(1)}^{* 0}(s)+\lambda_{2} q_{1(2)}^{* 0}(s)\right]\right\} /\left[s\left(s+\lambda_{1}+\lambda_{2}+\lambda_{12}\right)\right], \\
& q_{1(i)}^{* 0}(s)=\left[s+\lambda_{3-i}^{\prime}-\lambda_{3-i}^{\prime} e^{-s \tau}\right]\left[1-f_{i}^{*}\left(s+\lambda_{3-i}^{\prime}\right)\right] /\left[s\left(s+\lambda_{3-i}^{\prime}\right)\right]+f_{i}^{*}\left(s+\lambda_{3-i}^{\prime}\right) q_{2}^{* 0}(s), \\
& q_{0(i)}^{* 0}(s)=\left\{\left(1-e^{-s \tau}\right)+\left[e^{-s \tau} F_{i}(\tau)-f_{i}^{\dagger}(s)\right]\right\} / s+f_{i}^{\dagger}(s) q_{1(3-i)}^{* 0}(s), \\
& q_{2}^{* j}(s)=\left\{\lambda_{1} q_{1(1)}^{* j}(s)+\lambda_{2} q_{1(2)}^{* j}(s)+\lambda_{12}\left[\alpha_{1} q_{0(1)}^{* j-1}(s)\right.\right. \\
& \left.\left.\quad+\alpha_{2} q_{0(2)}^{* j-1}(s)-\delta_{1 j}\left(1-e^{-s \tau}\right) / s\right]\right\} /\left(s+\lambda_{1}+\lambda_{2}+\lambda_{12}\right) \quad(j \geqq 1), \\
& q_{1(i)}^{* 1}(s)=\beta_{i}^{*}(s ; \tau) q_{1(3-i)}^{* 0}(s)+f_{i}^{*}\left(s+\lambda_{3-i}^{\prime}\right) q_{2}^{* 1}(s)+\delta_{i}^{*}(s ; \tau), \\
& q_{1(i)}^{* j}(s)=\beta_{i}^{*}(s ; \tau) q_{1(3-i)}^{* j}(s)+f_{i}^{*}\left(s+\lambda_{3-i}^{\prime}\right) q_{2}^{* j}(s) \quad(j \geqq 2), \\
& q_{0(i)}^{* j}(s)=f_{i}^{\dagger}(s) q_{1(3-i)}^{* j}(s) .
\end{aligned}
$$

After considerable manipulation we have

$$
\begin{aligned}
Q_{1(i)}^{* 0}(s)= & q_{1(i)}^{* 0}(s) \\
= & {\left[\alpha_{3-i}^{*}(s) \gamma_{i}^{*}(s ; \tau)+\lambda_{3-i} f_{i}^{*}\left(s+\lambda_{3-i}^{\prime}\right) \gamma_{3-i}^{*}(s ; \tau)\right] /\left[\alpha_{1}^{*}(s)-\lambda_{2} f_{2}^{*}\left(s+\lambda_{1}^{\prime}\right)\right], } \\
Q_{0(i)}^{* 0}(s)= & \left.q_{0(i)}^{* 0}(s) \quad+\lambda_{i} f_{3-i}^{*}\left(s+\lambda_{i}^{\prime}\right) \gamma_{i}^{*}(s ; \tau)\right] /\left[\alpha_{1}^{*}(s)-\lambda_{2} f_{2}^{*}\left(s+\lambda_{1}^{\prime}\right)\right], \\
= & {\left[1-e^{-s \tau}-f_{i}^{\dagger}(s)+e^{-s \tau} F_{i}(\tau)\right] / s+f_{i}^{\dagger}(s)\left[\alpha_{i}^{*}(s) \gamma_{3-i}^{*}(s ; \tau)\right.} \\
Q_{2}^{* 0}(s)= & q_{2}^{* 0}(s) \quad \\
= & \left\{s+\lambda_{12}\left(1-e^{-s-}\right)+\sum_{j=1}^{2} \lambda_{j}\left[s+\lambda_{3-j}^{\prime}\left(1-e^{-s \tau}\right)\right]\left[1-f_{j}^{*}\left(s+\lambda_{3-j}^{\prime}\right)\right] /\left[s\left(s+\lambda_{3-j}^{\prime}\right)\right]\right\} \\
& /\left[\alpha_{1}^{*}(s)-\lambda_{2} f_{2}^{*}\left(s+\lambda_{1}^{\prime}\right)\right], \\
Q_{0(i)}^{* n}(s)= & {\left[1-f_{i}^{\dagger}(s)-e^{-s}\left(1-F_{i}(\tau)\right)\right] / s+f_{i}^{\dagger}(s) Q_{1(3-i)}^{* n}(s), } \\
Q_{2}^{* n}(s)= & \left\{1+\lambda_{1} Q_{1(1)}^{* n}(s)+\lambda_{2} Q_{1(2)}^{* n}(s)+\lambda_{12}\left[\alpha_{1} Q_{0(1)}^{* n-1}(s)+\alpha_{2} Q_{0(2)}^{* n-1}(s)\right]\right\} /\left(s+\lambda_{1}+\lambda_{2}+\lambda_{12}\right) \\
= & \left\{\sum_{j=1}^{2}\left[\lambda_{12} \alpha_{j} f_{j}^{\dagger}(s)+\lambda_{j} \beta_{j}^{*}(s ; \tau] Q_{1(3-j)}^{* n-1}(s)+\sum_{j=1}^{2} \lambda_{j} \varepsilon_{j}^{*}(s ; \tau)+\eta^{*}(s ; \tau)\right\}\right. \\
& /\left[\alpha_{1}^{*}(s)-\lambda_{2} f_{2}^{*}\left(s+\lambda_{1}^{\prime}\right)\right], \\
Q_{1(i)}^{* n}(s)= & \beta_{i}^{*}(s ; \tau) Q_{1(3-i)}^{* n-1}(s)+f_{i}^{*}\left(s+\lambda_{3-i}^{\prime}\right) Q_{2}^{* n}(s)+\varepsilon_{i}^{*}(s ; \tau) \\
= & {\left[\alpha_{1}^{*}(s)-\lambda_{2} f_{2}^{*}\left(s+\lambda_{1}^{\prime}\right)\right]^{-1}\left\{f_{j}^{*}\left(s+\lambda_{3-i}^{\prime}\right)\left[\lambda_{12} \alpha_{3-i} f_{3-i}^{\dagger}(s)+\lambda_{3-i} \beta_{i}^{*}(s ; \tau)\right] Q_{1(i)}^{* n-1}(s)\right.} \\
& +\left[\lambda_{12} \alpha_{i} f_{i}^{\dagger}(s) f_{i}^{*}\left(s+\lambda_{3-i}^{\prime}\right)+\beta_{i}^{*}(s ; \tau) \alpha_{3-i}^{*}(s)\right] Q_{1(3-i)}^{* n-1}(s)+f_{i}^{*}\left(s+\lambda_{3-i}^{\prime}\right) \eta^{*}(s ; \tau) \\
& \left.\left.+\alpha_{3-i}^{*}(s) \varepsilon_{i}^{*}(s ; \tau)+\lambda_{3-i} f_{i}^{*}\left(s+\lambda_{3-i}^{\prime}\right) \varepsilon_{3-i}^{*}(s ; \tau)\right\}, 2^{* * *}\right)
\end{aligned}
$$

We rewrite (21) as

$$
\begin{aligned}
& Q_{1(1)}^{* n}(s)=A_{1}^{*}(s ; \tau) Q_{1(1)}^{* n-1}(s)+B_{1}^{*}(s ; \tau) Q_{1(2)}^{* n-1}(s)+C_{1}^{*}(s ; \tau) \\
& Q_{1(2)}^{* n}(s)=A_{2}^{*}(s ; \tau) Q_{1(2)}^{* n-1}(s)+B_{2}^{*}(s ; \tau) Q_{1(1)}^{* n-1}(s)+C_{2}^{*}(s ; \tau)
\end{aligned}
$$

where

$$
\begin{aligned}
A_{i}^{*}(s ; \tau)= & f_{i}^{*}\left(s+\lambda_{3-i}^{\prime}\right)\left[\lambda_{12} \alpha_{3-i} f_{3-i}^{\dagger}(s)+\lambda_{3-i} \beta_{3-i}^{*}(s ; \tau)\right] /\left[\alpha_{1}^{*}(s)-\lambda_{2} f_{2}^{*}\left(s+\lambda_{1}^{\prime}\right)\right] \\
B_{i}^{*}(s ; \tau)= & {\left[\lambda_{12} \alpha_{i} f_{i}^{\dagger}(s) f_{i}^{*}\left(s+\lambda_{3-i}^{\prime}\right)+\alpha_{3-i}^{*}(s) \beta_{i}^{*}(s ; \tau)\right] /\left[\alpha_{1}^{*}(s)-\lambda_{2} f_{2}^{*}\left(s+\lambda_{1}^{\prime}\right)\right] } \\
C_{i}^{*}(s ; \tau)= & \left\{f_{i}^{*}\left(s+\lambda_{3-i}^{\prime}\right)\left[1+\lambda_{12} \sum_{j=1}^{2} \alpha_{j}\left[1-f_{j}^{\dagger}(s)-e^{-s}\left(1-F_{j}(\tau)\right)\right] / s\right]\right. \\
& \left.+\alpha_{3-i}^{*}(s) \varepsilon_{i}^{*}(s ; \tau)+\lambda_{3-i} f_{i}^{*}\left(s+\lambda_{3-i}^{\prime}\right) \varepsilon_{3-i}^{*}(s ; \tau)\right\} /\left[\alpha_{1}^{*}(s)-\lambda_{2} f_{2}^{*}\left(s+\lambda_{1}^{\prime}\right)\right] .
\end{aligned}
$$


In case when $\tau \neq 0$, we get $A_{1}^{*}(s ; \tau) A_{2}^{*}(s ; \tau) \neq B_{1}^{*}(s ; \tau) B_{2}^{*}(s ; \tau)$ and $\left[1-A_{1}^{*}(s ; \tau]\right.$ $\cdot\left[1-A_{2}^{*}(s ; \tau)\right]-B_{1}^{*}(s ; \tau) B_{2}^{*}(s ; \tau)>0 .^{\left(3^{* *}\right)}$ Therefore we obtain general solution

$$
\begin{aligned}
Q_{1(1)}^{* n}(s)= & E^{*}(s ; \tau) \rho^{*}(s ; \tau)^{n}+F^{*}(s ; \tau) \sigma^{*}(s ; \tau)^{n}+D_{1}^{*}(s ; \tau), \\
Q_{1(2)}^{* n}(s)= & E^{*}(s ; \tau)\left\{\left[\rho^{*}(s ; \tau)-A_{1}^{*}(s ; \tau)\right] / B_{1}^{*}(s ; \tau)\right\}^{n} \\
& +F^{*}(s ; \tau)\left\{\left[\sigma^{*}(s ; \tau)-A_{1}^{*}(s ; \tau)\right] / B_{1}^{*}(s ; \tau)\right\} \sigma^{*}(s ; \tau)^{n}+D_{2}^{*}(s ; \tau),
\end{aligned}
$$

where

$$
\begin{aligned}
& \rho^{*}(s ; \tau)=\left\{A_{1}^{*}(s ; \tau)+A_{2}^{*}(s ; \tau)\right. \\
& \left.+\sqrt{\left[A_{1}^{*}(s ; \tau)+A_{2}^{*}(s ; \tau)\right]^{2}-4\left[A_{1}^{*}(s ; \tau) A_{2}^{*}(s ; \tau)-B_{1}^{*}(s ; \tau) B_{2}^{*}(s ; \tau)\right]}\right\} / 2, \\
& \sigma^{*}(s ; \tau)=A_{1}^{*}(s ; \tau)+A_{2}^{*}(s ; \tau)-\rho^{*}(s ; \tau), \\
& D_{i}^{*}(s ; \tau)=\left\{B_{i}^{*}(s ; \tau) C_{3-i}^{*}(s ; \tau)+C_{i}^{*}(s ; \tau)\left[1-A_{3-i}^{*}(s ; \tau)\right]\right\} \\
& /\left\{\left[1-A_{1}^{*}(s ; \tau)\right]\left[1-A_{2}^{*}(s ; \tau)\right]-B_{1}^{*}(s ; \tau) B_{2}^{*}(s ; \tau)\right\}, \\
& E^{*}(s ; \tau)=\left\{\left[Q_{1(1)}^{* 0}(s)-D_{1}^{*}(s ; \tau)\right]\left[\sigma^{*}(s ; \tau)-A_{1}^{*}(s ; \tau)\right]\right. \\
& \left.-\left[Q_{1(2)}^{* 0}(s)-D_{2}^{*}(s ; \tau)\right] B_{1}^{*}(s ; \tau)\right\} /\left[\sigma^{*}(s ; \tau)-\rho^{*}(s ; \tau)\right], \\
& F^{*}(s ; \tau)=Q_{1(1)}^{* 0}(s)-D_{1}^{*}(s ; \tau)-E^{*}(s ; \tau) .
\end{aligned}
$$

Since we get $\left|\rho^{*}(s ; \tau)\right|<1$ and $\left|\sigma^{*}(s ; \tau)\right|<1^{\left(4^{* *}\right)}$, we have for limiting case as $n \rightarrow \infty$

$$
Q_{1(i)}^{* \infty}(s)=D_{i}^{*}(s ; \tau) \text {. }
$$

Using (17) and (19), we obtain $Q_{k(i)}^{n}(s)$ and $Q_{k(i)}^{\infty}(s)(k=2,0, i=1,2)$. Moreover we obtain the mean time to system failure, $\operatorname{MTSF}_{k(i)}^{n}$ and $\operatorname{MTSF}_{k(i)}^{\infty}$. For the limiting case as $n \rightarrow \infty$, we obtain after considerable manipulation ${ }^{\left(5^{* *}\right)}$

$$
\begin{aligned}
Q_{2}^{* \infty}(s)=p^{*}(s ; \tau)\left\{1+\sum_{j=1}^{2} \frac{\lambda_{j}}{s+\lambda_{j}^{\prime}}+\lambda_{12} \cdot \sum_{j=1}^{2} \alpha_{j}\right. & {\left[\frac{1-e^{-s \tau}}{s}+\frac{f_{j}^{\dagger}(s)}{s+\lambda_{3-j}^{\prime}}-F_{j}^{\dagger}(s)\right] } \\
& \left.-\sum_{j=1}^{2} \frac{N_{j}^{*}(s ; \tau)}{\left(s+\lambda_{3-j}^{\prime}\right)\left[1-\beta_{1}^{*}(s ; \tau) \beta_{2}^{*}(s ; \tau)\right]}\right\}
\end{aligned}
$$

where

$$
\begin{aligned}
p^{*}(s ; \tau)= & \left\{s+\lambda_{1}+\lambda_{2}+\lambda_{12}-\frac{\sum_{j=1}^{2} f_{j}\left(s+\lambda_{3-j}^{\prime}\right)\left[k_{j}^{*}(s ; \tau) \beta_{3-j}^{*}(s ; \tau)+k_{3-j}^{*}(s ; \tau)\right]}{1-\beta_{1}^{*}(s ; \tau) \beta_{2}^{*}(s ; \tau)}\right\}^{-1}, \\
N_{j}^{*}(s ; \tau)= & \frac{\lambda_{3-j}^{\prime}}{s}\left\{k_{3-j}^{*}(s ; \tau)-k_{j}^{*}(s ; \tau)+\left[k_{j}^{*}(s ; \tau) \beta_{3-j}^{*}(s ; \tau)-k_{3-j}^{*}(s ; \tau) \beta_{j}^{*}(s ; \tau)\right]\right\} \\
& +\left[k_{j}^{*}(s ; \tau) \beta_{3-j}^{*}(s ; \tau)+k_{3-j}^{*}(s ; \tau)\right]\left\{f_{j}^{*}\left(s+\lambda_{3-j}^{\prime}\right)+\lambda_{3-j}^{\prime}\left[\frac{e^{-s \tau}-1}{s}+F_{j}^{\dagger}(s)\right]\right\}
\end{aligned}
$$

(28) coincides with formula (22) in Fukuta and Kodama [1]. Therefore all the results in this paper for the limiting case coincide with the results in [1]. Hence we cite only the finite case.

Especially when $\tau=0$, we have $\beta_{i}^{*}(s ; 0)=f_{i}^{\dagger}(s)=0$ and $A_{i}^{*}(s ; 0)=B_{i}^{*}(s ; 0)=0$. Hence we have

$$
\begin{aligned}
Q_{1(i)}^{* n}(s)=D_{i}^{*}(s ; \tau) & =C_{i}^{*}(s ; \tau)=\left\{f_{i}^{*}\left(s+\lambda_{3-i}^{\prime}\right)+\alpha_{3-i}^{*}(s)\left[1-f_{i}^{*}\left(s+\lambda_{3-i}^{\prime}\right)\right] /\left(s+\lambda_{3-i}^{\prime}\right)\right. \\
& \left.+\lambda_{3-i} f_{i}^{*}\left(s+\lambda_{3-i}^{\prime}\right)\left[1-f_{3-i}^{*}\left(s+\lambda_{i}^{\prime}\right)\right] /\left(s+\lambda_{i}^{\prime}\right)\right\} /\left[\alpha_{1}^{*}(s)-\lambda_{2} f_{2}^{*}\left(s+\lambda_{1}^{\prime}\right)\right]
\end{aligned}
$$

for all $n$, 


$$
Q_{2}^{* n}(s)=\left\{1+\sum_{j=1}^{2} \lambda_{j}\left[1-f_{j}\left(s+\lambda_{3-j}^{\prime}\right)\right] /\left(s+\lambda_{3-j}^{\prime}\right)\right\} /\left[\alpha_{1}^{*}(s)-\lambda_{2} f_{2}^{*}\left(s+\lambda_{1}^{\prime}\right)\right] \quad \text { for all } n
$$

Above formulae coincide the Laplace transform of probability that the major breakdown does not occur during the time interval $(0, t)$, given that the system starts in $E_{1}(i)$ or $E_{2}$ at $t=0$.

For two identical unit model, the formula do not depend on $i$, so we define $q_{1(i)}^{* j}(s)=q_{1}^{* j}(s), Q_{1(i)}^{* j}(s)=Q_{1}^{* j}(s), \cdots$, so on. Noting that $\rho^{*}(s ; \tau)=A^{*}(s ; \tau)+B^{*}(s ; \tau)$, $\sigma^{*}(s ; \tau)=A^{*}(s ; \tau)-B^{*}(s ; \tau), E^{*}(s ; \tau)=Q_{1}^{* 0}(s)-D^{*}(s ; \tau)$ and $F^{*}(s ; \tau)=0$, we have

$$
\begin{aligned}
Q_{1}^{* n}(s)=\{ & \left.\frac{\lambda_{12} f^{\dagger}(s) f^{*}\left(s+\lambda^{\prime}\right)+\left(s+2 \lambda+\lambda_{12}\right) \beta^{*}(s ; \tau)}{\alpha^{*}(s)-\lambda f^{*}\left(s+\lambda^{\prime}\right)}\right\}^{n}\left\{\frac{\left(s+2 \lambda+\lambda_{12}\right) \gamma^{*}(s ; \tau)}{\alpha^{*}(s)-\lambda f^{*}\left(s+\lambda^{\prime}\right)}\right\} \\
+ & \left\{1-\left[\frac{\lambda_{12} f^{\dagger}(s) f^{*}\left(s+\lambda^{\prime}\right)+\left(s+2 \lambda+\lambda_{12}\right) \beta^{*}(s ; \tau)}{\alpha^{*}(s)-\lambda f^{*}\left(s+\lambda^{\prime}\right)}\right]^{n}\right\} \\
& \cdot\left\{\frac{f^{*}\left(s+\lambda^{\prime}\right)\left[1+\lambda_{12}\left[1-f^{\dagger}(s)-e^{-s *}(1-F(\tau))\right] / s\right]+\left(s+2 \lambda+\lambda_{12}\right) \varepsilon(s ; \tau)}{\left(s+2 \lambda+\lambda_{12}\right)\left[1-\beta^{*}(s ; \tau)\right]-\left[2 \lambda+\lambda_{12} f^{\dagger}(s)\right] f^{*}\left(s+\lambda^{\prime}\right)}\right\}, \quad(32) \\
Q_{2}^{* n}(s)= & \frac{1+\left[\lambda_{12} f^{\dagger}(s)+2 \lambda \beta^{*}(s ; \tau)\right] Q_{1}^{* n-1}(s)+2 \lambda \varepsilon^{*}(s ; \tau)+\lambda_{12}\left[1-f^{\dagger}(s)-e^{-s z}(1-F(\tau))\right] / s}{\alpha^{*}(s)-\lambda f^{*}\left(s+\lambda^{\prime}\right)}, \\
\operatorname{MTSF}_{1}^{n}= & \left\{\frac{\lambda_{12} F(\tau) f^{*}\left(\lambda^{\prime}\right)+\left(2 \lambda+\lambda_{12}\right) \beta^{*}(0 ; \tau)}{\lambda_{12}+2 \lambda\left[1-f^{*}\left(\lambda^{\prime}\right)\right]}\right\}^{n}\left\{\frac{\left(2 \lambda+\lambda_{12}\right) \gamma^{*}(0 ; \tau)}{\lambda_{12}+2 \lambda\left[1-f^{*}\left(\lambda^{\prime}\right)\right]}\right\} \\
& +\left\{1-\left[\frac{\lambda_{12} F(\tau) f^{*}\left(\lambda^{\prime}\right)+\left(2 \lambda+\lambda_{12}\right) \beta^{*}(0 ; \tau)}{\lambda_{12}+2 \lambda\left[1-f^{*}\left(\lambda^{\prime}\right)\right]}\right]^{n}\right\} \\
& \cdot\left\{\frac{f^{*}\left(\lambda^{\prime}\right)\left[1+\lambda_{12}\left(\tau-\int_{0}^{\tau} F(t) d t\right)\right]+\left(2 \lambda+\lambda_{12}\right) \varepsilon^{*}(0 ; \tau)}{\left(2 \lambda+\lambda_{12}\right)\left[1-\beta^{*}(0 ; \tau)\right]-\left[2 \lambda+\lambda_{12} F(\tau)\right] f^{*}\left(\lambda^{\prime}\right)}\right\}, \\
\operatorname{MTSF}_{2}^{n}= & \frac{1+\left[\lambda_{12} F(\tau)+2 \lambda \beta^{*}(0 ; \tau)\right] \operatorname{MTSF} F_{1}^{n-1}+2 \lambda \varepsilon^{*}(0 ; \tau)+\lambda_{12}\left[\tau-\int_{0}^{\tau} F(t) d t\right]}{\lambda_{12}+2 \lambda\left[1-f^{*}(\lambda)\right]} .
\end{aligned}
$$

Especially when $F(t)=1-\exp (-\mu t)$, we have $\mathrm{e}^{\left(6^{* *}\right)}$

$$
\begin{aligned}
\operatorname{MTSF}_{1}^{n}= & {\left[1-e^{-\mu \tau}\right]^{n}\left[\tau+\frac{2 \lambda+\lambda_{12}+\mu}{2 \lambda \lambda^{\prime}+\lambda_{12}\left(\lambda^{\prime}+\mu\right)}\right] } \\
& +\left[1-\left(1-e^{-\mu \tau}\right)^{n}\right]\left\{\left[\frac{1}{\mu}+\frac{2 \lambda+\lambda_{12}+\mu}{2 \lambda \lambda^{\prime}+\lambda_{12}\left(\lambda^{\prime}+\mu\right)}\right] e^{-\mu \tau}-\frac{1}{\mu}\right\}, \\
\operatorname{MTSF}_{2}^{n}= & \frac{\left(2 \lambda+\lambda^{\prime}+\mu\right)+\left[2 \lambda \lambda^{\prime}+\lambda_{12}\left(\lambda^{\prime}+\mu\right)\right]\left(1-e^{-\mu \tau}\right)\left[\operatorname{MTSF}_{1}^{n-1}+\frac{1}{\mu}\right]}{2 \lambda \lambda^{\prime}+\lambda_{12}\left(\lambda^{\prime}+\mu\right)} .
\end{aligned}
$$

The improvement factor $I^{n}=\lambda \operatorname{MTSF}_{2}^{n}$ (the ratio of the MTSF for this system to one unit system without repair) are show in Table 1 for the case $\lambda_{12}=0$ and $\lambda^{\prime}=\lambda$.

\section{Acknowledgements}

Our thanks are due to Professor J. Gani, Sheffield University, for his interest in this work. The authors also wish to thank Dr. S. Takamatsu, Osaka University and Mr. H. Nakamichi, Otemon Gakuin University, Japan, for their suggestions. 
Table 1 The improvement factor $I^{n}$

\begin{tabular}{|c|c|c|c|c|c|c|c|c|c|}
\hline \multirow{2}{*}{$\rho(=\mu / \lambda)$} & \multirow{2}{*}{$n$} & \multicolumn{8}{|c|}{$m(=\mu \tau)$} \\
\hline & & \multicolumn{2}{|l|}{$10^{-2}$} & \multicolumn{2}{|l|}{$10^{-1}$} & \multicolumn{2}{|l|}{1} & \multicolumn{2}{|l|}{10} \\
\hline \multirow{7}{*}{100} & 0 & $0.5150 \mathrm{E}$ & 02 & $0.5150 \mathrm{E}$ & 02 & $0.5151 \mathrm{E}$ & 02 & $0.5160 \mathrm{E}$ & 02 \\
\hline & 1 & $0.5201 \mathrm{E}$ & 02 & $0.5635 \mathrm{E}$ & 02 & $0.8375 \mathrm{E}$ & 02 & $0.1026 \mathrm{E}$ & 03 \\
\hline & 3 & $0.5201 \mathrm{E}$ & 02 & $0.5686 \mathrm{E}$ & 02 & $0.1170 \mathrm{E}$ & 03 & $0.2046 \mathrm{E}$ & 03 \\
\hline & 5 & $0.5201 \mathrm{E}$ & 02 & $0.5686 \mathrm{E}$ & 02 & $0.1303 \mathrm{E}$ & 03 & $0.3066 \mathrm{E}$ & 03 \\
\hline & 10 & $0.5201 \mathrm{E}$ & 02 & $0.5686 \mathrm{E}$ & 02 & $0.1383 \mathrm{E}$ & 03 & $0.5616 \mathrm{E}$ & 03 \\
\hline & 100 & $0.5201 \mathrm{E}$ & 02 & $0.5686 \mathrm{E}$ & 02 & $0.1391 \mathrm{E}$ & 03 & $0.5141 \mathrm{E}$ & 04 \\
\hline & $\infty$ & $0.5201 \mathrm{E}$ & 02 & $0.5686 \mathrm{E}$ & 02 & $0.1391 \mathrm{E}$ & 03 & $0.1124 \mathrm{E}$ & 07 \\
\hline
\end{tabular}

\section{Appendix}

DERIYATION OF $\left(1^{* *}\right)$

$$
\begin{aligned}
& q_{1(i)}^{* j}(s)= \int_{0}^{\infty} e^{-s t} q_{1(i)}^{j}(t) d t, \quad j \geqq 1 \\
&= \int_{\tau}^{\infty} e^{-s T} d T \int_{0}^{T-\tau} d x \int_{0}^{\tau} \lambda_{3-i}^{\prime} e^{-\lambda_{3-i}^{\prime} x} f_{i}(x+y) q_{1(3-i)}^{j-1}(T-x-y) d y \\
&+\int_{\tau}^{\infty} e^{-s T} d T \int_{0}^{T-\tau} e^{-\lambda_{3-i}^{\prime} x} f_{i}(x) q_{2}^{j}(T-x) d x \\
&= \int_{0}^{\tau} d y \int_{0}^{\infty} \lambda_{3-i}^{\prime} e^{-\lambda_{3-i}^{\prime} x} f_{i}(x+y) d x \int_{x+\tau}^{\infty} e^{-s T} q_{1(3-i)}^{j-1}(T-x-y) d T \\
&+\int_{0}^{\infty} e^{-\lambda_{3-i}^{\prime} x} f_{i}(x) d x \int_{x+\tau}^{\infty} e^{-s T} q_{2}^{j}(T-x) d T \\
&= \int_{0}^{\tau} e^{-s y} d y \int_{0}^{\infty} \lambda_{3-i}^{\prime} e^{-\left(s+\lambda_{3-i}^{\prime}\right) x} f_{i}(x+y) d x \int_{\tau-y}^{\infty} e^{-s u} q_{1(3-i)}^{j-1}(u) d u \\
&+\int_{0}^{\infty} e^{-\lambda_{3-i}^{\prime}} f_{i}(x) d x \int_{\tau}^{\infty} e^{-s(x+u)} q_{2}^{j}(u) d u \\
&= \int_{0}^{\tau} e^{-s y} d y \int_{0}^{\infty} \lambda_{3-i}^{\prime} e^{-\left(s+\lambda_{3-i}^{\prime}\right) x} f_{i}(x+y) d x\left[q_{1(3-i)}^{* j-1}(s)-\int_{0}^{\tau-y} e^{-s u} q_{1(3-i)}^{j-1}(u) d u\right] \\
&+\int_{0}^{\infty} e^{-\left(s+\lambda_{3-i}^{\prime}\right) x} f_{i}(x) d x\left[\int_{0}^{\infty} e^{-s u} q_{2}^{j}(u) d u-\int_{0}^{\tau} e^{-s u} q_{2}^{j}(u) d u\right] \\
&= \int_{0}^{\tau} e^{-s y} d y \int_{0}^{\infty} \lambda_{3-i}^{\prime} e^{-\left(s+\lambda_{3-i}^{\prime}\right) x} f_{i}(x+y) d x\left\{q_{1(3-i)}^{* j-1}(s)-\delta_{1 j}\left[1-e^{-s(\tau-y)}\right] / s\right\} \\
&+f_{i}^{*}\left(s+\lambda_{3-i}^{\prime}\right) q_{2}^{* j}(s) . \\
& \int_{0}^{\tau} e^{-s y} d y \int_{0}^{\infty} \lambda_{3-i}^{\prime} e^{-\left(s+\lambda_{3-i}^{\prime}\right) x} f_{i}(x+y)\left[q_{1(3-i)}^{* j-1}(s)-\frac{1}{s}+\frac{1}{s} e^{-s(\tau-y)}\right] d x \\
&= \beta_{i}^{*}(s ; \tau)\left[q_{1(3-i)}^{* j-1}(s)-\frac{1}{s}\right]+\frac{1}{s} e^{-s \tau} \int_{0}^{\tau} d y \int_{0}^{\infty} \lambda_{3-i}^{\prime} e^{-\left(s+\lambda_{3-i}^{\prime}\right) x} f_{i}(x+y) d x \\
& j=1=
\end{aligned}
$$

(i) $j=1$

where 


$$
\begin{aligned}
& \beta_{i}^{*}(s ; \tau)= \int_{0}^{\tau} e^{-s y} d y \int_{0}^{\infty} \lambda_{3-i}^{\prime} e^{-\left(s+\lambda_{3-i}^{\prime}\right) x} f_{i}(x+y) d x \\
&= \int_{0}^{\tau} e^{-s y} d y \int_{y}^{\infty} \lambda_{3-i}^{\prime} e^{-\left(s+\lambda_{3-i}^{\prime}\right)(v-y)} f_{i}(v) d v \\
&= \int_{0}^{\tau} \lambda_{3-i}^{\prime} e^{-\lambda_{3-i}^{\prime} y} d y\left[\int_{0}^{\infty} e^{-\left(s+\lambda_{3-i}^{\prime}\right) v} f_{i}(v) d v-\int_{0}^{y} e^{-\left(s+\lambda_{3-i}^{\prime}\right) v} f_{i}(v) d v\right] \\
&=\left(e^{\lambda_{3-i}^{\prime} \tau}-1\right) f_{i}^{*}\left(s+\lambda_{3-i}^{\prime}\right)-\int_{0}^{\tau} e^{-\left(s+\lambda_{3-i}^{\prime}\right) v} f_{i}(v) d v \int_{v}^{\tau} \lambda_{3-1}^{\prime} e^{\lambda_{3-i}^{\prime} y} d y \\
&=\left(e^{\lambda_{3-i}^{\prime}}-1\right) f_{i}^{*}\left(s+\lambda_{3-i}^{\prime}\right)-e^{\lambda_{3-i}^{\tau}} f_{i}^{\dagger}\left(s+\lambda_{3-i}^{\prime}\right)+f_{i}^{\dagger}(s) \\
& \int_{0}^{\tau} d y \int_{0}^{\infty} \lambda_{3-i}^{\prime} e^{-\left(s+\lambda_{3-i}^{\prime}\right) x} f_{i}(x+y) d x \\
&=\int_{0}^{\tau} d y \int_{y}^{\infty} \lambda_{3-i}^{\prime} e^{-\left(s+\lambda_{3-i}^{\prime}\right)(v-y)} f_{i}(v) d v \\
&= \int_{0}^{\tau} \lambda_{3-i}^{\prime} e^{\left(s+\lambda_{3-i}^{\prime}\right) y} d y\left[\int_{0}^{\infty} e^{-\left(s+\lambda_{3-i}^{\prime}\right) v} f_{i}(v) d v-\int_{0}^{y} e^{-\left(s+\lambda_{3-i}^{\prime}\right) v} f_{i}(v) d v\right] \\
&= \lambda_{3-i}^{\prime} f_{i}^{*}\left(s+\lambda_{3-i}^{\prime}\right)\left[e^{\left(s+\lambda_{3-i}^{\prime}\right) \tau}-1\right] /\left(s+\lambda_{3-i}^{\prime}\right) \\
&-\int_{0}^{\tau} e^{-\left(s+\lambda_{3-i}^{\prime}\right) v} f_{i}(v) d v \int_{v}^{\tau} \lambda_{3-i}^{\prime} e^{\left(s+\lambda_{3-i}^{\prime}\right) y} d y \\
&= \frac{\lambda_{3-i}^{\prime}}{s+\lambda_{3-i}^{\prime}}\left\{f_{i}^{*}\left(s+\lambda_{3-i}^{\prime}\right)\left[e^{\left(s+\lambda_{3-i}^{\prime}\right) \tau}-1\right]-f_{i}^{\dagger}\left(s+\lambda_{3-i}^{\prime}\right) e^{\left(s+\lambda_{3-i}^{\prime}\right) \tau}+F_{i}(\tau)\right\} .
\end{aligned}
$$

Hence we have

$$
\begin{aligned}
q_{1(i)}^{* 1}(s)= & \beta_{i}(s ; \tau)\left[q_{1(3-i)}^{* 0}(s)-\frac{1}{s}\right] \\
& +\frac{\lambda_{3-i}^{\prime} e^{-s \tau}}{s\left(s+\lambda_{3-i}^{\prime}\right)}\left\{f_{i}^{*}\left(s+\lambda_{3-i}^{\prime}\right)\left[e^{\left(s+\lambda_{3-i}^{\prime}\right) \tau}-1\right]-f_{i}^{\dagger}\left(s+\lambda_{3-i}^{\prime}\right) e^{\left(s+\lambda_{3-i}^{\prime}\right) \tau}+F_{i}(\tau)\right\} \\
& +f_{i}^{*}\left(s+\lambda_{3-i}^{\prime}\right) q_{2}^{* 1}(s) \\
= & \beta_{i}(s ; \tau) q_{1(3-i)}^{* 0}(s)+f_{i}^{*}\left(s+\lambda_{3-i}^{\prime}\right) q_{2}^{* 1}(s)+\delta_{i}^{*}(s ; \tau) .
\end{aligned}
$$

(ii) $j \geqq 2$

$$
\begin{aligned}
q_{1(i)}^{* j}(s) & =\int_{0}^{\tau} e^{-s y} d y \int_{0}^{\infty} \lambda_{3-i}^{\prime} e^{-\left(s+\lambda_{3-i}^{\prime}\right) x} f_{i}(x+y) d x \cdot q_{1(3-i)}^{* j-1}(s)+f_{i}^{*}\left(s+\lambda_{3-i}^{\prime}\right) q_{2}^{* j}(s) \\
& =\beta_{i}^{*}(s ; \tau) q_{1(3-i)}^{* j-1}(s)+f_{i}^{*}\left(s+\lambda_{3-i}^{\prime}\right) q_{2}^{* j}(s) .
\end{aligned}
$$

Similary we have the formulae (7)-(10) and (13).

\section{DeRIVATIONS OF $\left(2^{* *}\right)$}

Substituting (17) and (18) into (20) and arranging, we have

$Q_{1(1)}^{* n}(s)\left[s+\lambda_{1}+\lambda_{2}+\lambda_{12}-\lambda_{1} f_{1}^{*}\left(s+\lambda_{2}^{\prime}\right)\right]$

$$
\begin{gathered}
=\lambda_{2} f_{1}^{*}\left(s+\lambda_{2}^{\prime}\right) Q_{1(2)}^{* n}(s)+f_{1}^{*}\left(s+\lambda_{2}^{\prime}\right)\left\{1+\lambda_{12} \cdot \sum_{j=1}^{2} \alpha_{j}\left[\left[1-f_{j}^{\dagger}(s)-e^{-s \tau}\left(1-F_{j}(\tau)\right)\right] / s\right.\right. \\
\left.\left.+f_{j}(s) Q_{1(3-j)}^{* n-1}(s)\right]\right\},
\end{gathered}
$$




$$
\begin{aligned}
& Q_{1(2)}^{* n}(s)\left[s+\lambda_{1}+\lambda_{2}+\lambda_{12}-\lambda_{2} f_{2}^{*}\left(s+\lambda_{1}^{\prime}\right)\right] \\
& =\lambda_{1} f_{2}^{*}\left(s+\lambda_{1}^{\prime}\right) Q_{1(1)}^{* n}(s)+f_{2}^{*}\left(s+\lambda_{1}^{\prime}\right)\left\{1+\lambda_{12} \cdot \sum_{j=1}^{2} \alpha_{j}\left[\left[1-f_{j}^{\dagger}(s)-e^{-s \tau}\left(1-F_{j}(\tau)\right)\right] / s\right.\right. \\
& \left.\left.\quad+f_{j}^{\dagger}(s) Q_{1(3-j)}^{* n-1}(s)\right]\right\} .
\end{aligned}
$$

From the above equations we have (21) and (22).

Derivation OF $\left(3^{* *}\right)$

After simple calculation, we have the following inequalities

$$
\begin{aligned}
\beta_{i}^{*}(s ; \tau) & \geqq\left(e^{\lambda_{3}-i=}-1\right)\left[f_{i}^{*}\left(s+\lambda_{3-i}\right)-f_{i}^{\dagger}\left(s+\lambda_{3-i}\right)\right] \\
& \geqq 0,
\end{aligned}
$$

the equality is true when $\tau=0$

$$
\begin{aligned}
& 1-f_{i}^{*}\left(s+\lambda_{3-i}^{\prime}\right)-\beta_{i}^{*}(s ; \tau) \\
& =1-e^{\lambda_{3-i}^{\prime} \tau} \int_{\tau}^{\infty} e^{-\left(s+\lambda_{3-i}^{\prime}\right) x} f_{i}(x) d x-f_{i}^{\dagger}(s) \\
& >1-e^{\lambda_{3-i}^{\prime} \tau} \cdot e^{-\left(s+\lambda_{3-i}^{\prime}\right) \tau}\left[1-F_{i}(\tau)\right]-F_{i}(\tau) \\
& \geqq 1-\left[1-F_{i}(\tau)\right]-F_{i}(\tau) \\
& =0 \text {. } \\
& 1-f_{i}^{*}\left(s+\lambda_{3-i}^{\prime}\right)-\beta_{i}^{*}(s ; \tau)\left[\beta_{3-i}^{*}(s ; \tau)+f_{3-i}^{*}\left(s+\lambda_{3}^{\prime}\right)\right] \\
& >1-f_{i}^{*}\left(s+\lambda_{3-i}^{\prime}\right)-\beta_{i}(s ; \tau) \quad \text { by }(39) \\
& >0 \text {. by }(39) \\
& 1-\beta_{i}^{*}(s ; \tau) \beta_{3-i}^{*}(s ; \tau)-\alpha_{3-i} f_{3-i}^{\dagger}(s)\left[f_{i}^{*}\left(s+\lambda_{3-i}^{\prime}\right)+f_{3-i}^{*}\left(s+\lambda_{i}^{\prime}\right) \beta_{i}^{*}(s ; \tau)\right] \\
& -\alpha_{i} f_{i}^{\dagger}(s)\left[f_{3-i}^{*}\left(s+\lambda_{i}^{\prime}\right)+f_{i}^{*}\left(s+\lambda_{3-i}^{\prime}\right) \beta_{3-i}^{*}(s ; \tau)\right] \\
& >1-\alpha_{i}\left\{f_{3-i}^{*}\left(s+\lambda_{i}^{\prime}\right)+\beta_{3-i}^{*}(s ; \tau)\left[f_{i}^{*}\left(s+\lambda_{3-i}^{\prime}\right)+\beta_{i}^{*}(s ; \tau)\right]\right\} \\
& -\alpha_{3-i}\left\{f_{i}^{*}\left(s+\lambda_{3-i}^{\prime}\right)+\beta_{i}^{*}(s ; \tau)\left[f_{3-i}^{*}\left(s+\lambda_{i}^{\prime}\right)+\beta_{3-i}^{*}(s ; \tau)\right]\right\} \\
& >1-\alpha_{i}-\alpha_{3-i} \quad \text { by }(40) \\
& =0 \text {. }
\end{aligned}
$$

It is clear that

$$
1-\beta_{i}^{*}(s ; \tau) \beta_{3-i}^{*}(s ; \tau)>0, \quad \alpha_{1}^{*}(s)-\lambda_{2} f_{2}^{*}\left(s+\lambda_{1}^{\prime}\right)>0 .
$$

Also from (23) we have

$$
\begin{aligned}
{\left[1-A_{1}^{*}\right.} & (s ; \tau)]\left[1-A_{2}^{*}(s ; \tau)\right]-B_{1}^{*}(s ; \tau) B_{2}^{*}(s ; \tau) \\
= & {\left[\alpha_{1}^{*}(s)-\lambda_{2} f_{2}^{*}\left(s+\lambda_{1}^{\prime}\right)\right]^{-1}\left\{s\left[1-\beta_{1}^{*}(s ; \tau) \beta_{2}^{*}(s ; \tau)\right]\right.} \\
& +\lambda_{1}\left[1-\beta_{1}^{*}(s ; \tau) \beta_{2}^{*}(s ; \tau)-f_{1}^{*}\left(s+\lambda_{2}^{\prime}\right)-f_{2}^{*}\left(s+\lambda_{1}^{\prime}\right) \beta_{1}^{*}(s ; \tau)\right] \\
& +\lambda_{2}\left[1-\beta_{1}^{*}(s ; \tau) \beta_{2}^{*}(s ; \tau)-f_{2}^{*}\left(s+\lambda_{1}\right)-f_{1}^{*}\left(s+\lambda_{2}^{\prime}\right) \beta_{2}^{*}(s ; \tau)\right] \\
& +\lambda_{12}\left[1-\beta_{1}^{*}(s ; \tau) \beta_{2}^{*}(s ; \tau)-\alpha_{2} f_{2}^{\dagger}(s)\left[f_{1}^{*}\left(s+\lambda_{2}^{\prime}\right)+f_{2}^{*}\left(s+\lambda_{1}^{\prime}\right) \beta_{1}^{*}(s ; \tau)\right]\right. \\
& \left.\left.-\alpha_{1} f_{1}^{\dagger}(s)\left[f_{2}^{*}\left(s+\lambda_{1}^{\prime}\right)+f_{1}^{*}\left(s+\lambda_{2}^{\prime}\right) \beta_{2}^{*}(s ; \tau)\right]\right]\right\} .
\end{aligned}
$$


Therefore from (35)-(40) we have

$$
\left[1-A_{1}^{*}(s ; \tau)\right]\left[1-A_{2}^{*}(s ; \tau)\right]-B_{1}^{*}(s ; \tau) B_{2}^{*}(s ; \tau)>0 .
$$

DERIVATION OF $\left(4^{* *}\right)$

From the definition and (35) we have

$$
\begin{aligned}
& f_{i}^{*}\left(s+\lambda_{3-i}^{\prime}\right)\left[\lambda_{12} \alpha_{3-i} f_{3-i}^{\dagger}(s)+\lambda_{3-i} \beta_{3-i}^{*}(s ; \tau)\right] \geqq 0, \\
& {\left[\lambda_{12} \alpha_{i} f_{i}^{\dagger}(s) f_{i}^{*}\left(s+\lambda_{3-i}^{\prime}\right)+\alpha_{3-i}^{*}(s) \beta_{i}^{*}(s ; \tau)\right] \geqq 0,}
\end{aligned}
$$

the equalities are true when $\tau=0$.

Also we have

$$
\begin{aligned}
& \alpha_{1}^{*}(s)-\lambda_{2} f_{2}^{*}\left(s+\lambda_{1}^{\prime}\right)-f_{i}^{*}\left(s+\lambda_{3-i}^{\prime}\right)\left[\lambda_{12} \alpha_{3-i} f_{3-i}^{\dagger}(s)+\lambda_{3-i} \beta_{3-i}^{*}(s ; \tau)\right] \\
& >\alpha_{1}^{*}(s)-\lambda_{2} f_{2}^{*}\left(s+\lambda_{1}^{\prime}\right)-\left[\lambda_{12}+\lambda_{3-i} \beta_{3-i}^{*}(s ; \tau)\right] \\
& =s+\lambda_{1}\left[1-f_{1}^{*}\left(s+\lambda_{2}^{\prime}\right)-\delta_{i 2} \beta_{3-i}^{*}(s ; \tau)\right]+\lambda_{2}\left[1-f_{1}^{*}\left(s+\lambda_{1}^{\prime}\right)-\delta_{i 1} \beta_{3-i}^{*}(s ; \tau)\right] \\
& >0, \quad \text { by } \quad(40) \\
& \alpha_{1}^{*}(s)-\lambda_{2} f_{2}^{*}\left(s+\lambda_{1}^{\prime}\right)-\lambda_{12} \alpha_{i} f_{i}^{\dagger}(s) f_{2}^{*}\left(s+\lambda_{3-i}^{\prime}\right)-\alpha_{3-i}^{*}(s) \beta_{i}^{*}(s ; \tau) \\
& \geqq s\left[1-\beta_{i}^{*}(s ; \tau)\right]+\lambda_{1}\left[1-f_{1}^{*}\left(s+\lambda_{2}^{\prime}\right)-\beta_{i}^{*}(s ; \tau)+\delta_{i 2} \beta_{3-i}^{*}(s ; \tau)\right] \\
& \quad+\lambda_{2}\left[1-f_{2}^{*}\left(s+\lambda_{1}^{\prime}\right)-\beta_{i}^{*}(s ; \tau)+\delta_{i 1} \beta_{3-i}^{*}(s ; \tau)\right]+\lambda_{12}\left[1-f_{i}^{*}\left(s+\lambda_{3-i}^{\prime}\right)-\beta_{i}^{*}(s ; \tau)\right] \\
& >0 . \quad \text { by }(40)
\end{aligned}
$$

From (44)-(47) we have

$$
1>A_{i}^{*}(s ; \tau) \geqq 0, \quad 1>B_{i}^{*}(s ; \tau) \geqq 0 .
$$

Since we have $A_{1}^{*}(s ; \tau)+A_{2}^{*}(s ; \tau)>0,(\tau \neq 0)$, to prove $\left|\rho^{*}(s ; \tau)\right|<1$ and $\left|\sigma^{*}(s ; \tau)\right|<1$ it suffices to show the following inequalities

$$
1>A_{1}^{*}(s ; \tau) A_{2}^{*}(s ; \tau)-B_{1}^{*}(s ; \tau) B_{2}^{*}(s ; \tau)>A_{1}^{*}(s ; \tau)+A_{2}^{*}(s ; \tau)-1 .
$$

The inequality on the right becomes

$$
\left[1-A_{1}^{*}(s ; \tau)\right]\left[1-A_{2}^{*}(s ; \tau)\right]-B_{1}^{*}(s ; \tau) B_{2}^{*}(s ; \tau)>0
$$

which is true by (43).

On the other hand, the inequality on the left is clear from $(48),(\tau \neq 0)$. Hence we have $\left|\rho^{*}(s ; \tau)\right|<1$ and $\left|\sigma^{*}(s ; \tau)\right|<1$.

DERIVATION OF $\left(5^{* *}\right)$.

We obtain after considerable manipulation

$$
\begin{aligned}
& {\left[1-A_{1}^{*}(s ; \tau)\right]\left[1-A_{2}^{*}(s ; \tau)\right]-B_{1}^{*}(s ; \tau) B_{2}^{*}(s ; \tau)} \\
& \quad=\left[\alpha_{1}^{*}(s)-\lambda_{2} f_{2}^{*}\left(s+\lambda_{1}^{\prime}\right)\right]^{-1}\left\{\left(s+\lambda_{1}+\lambda_{2}+\lambda_{12}\right)\left[1-\beta_{1}^{*}(s ; \tau) \beta_{2}^{*}(s ; \tau)\right]\right. \\
& \left.\quad-\sum_{j=1}^{2} f_{j}^{*}\left(s+\lambda_{3-j}^{\prime}\right) \cdot\left[k_{3-j}^{*}(s ; \tau)+\beta_{3-j}^{*}(s ; \tau) k_{j}^{*}(s ; \tau)\right]\right\}
\end{aligned}
$$




$$
\begin{aligned}
& B_{i}^{*}(s ; \tau) C_{3-i}^{*}(s ; \tau)+C_{i}^{*}(s ; \tau)\left[1-A_{3-i}^{*}(s ; \tau)\right] \\
&=\left[\alpha_{1}^{*}(s)-\lambda_{2} f_{2}^{*}\left(s+\lambda_{1}^{\prime}\right)\right]^{-1}\left\{\eta^{*}(s ; \tau)\left[f_{1}^{*}\left(s+\lambda_{3-i}^{\prime}\right)+\beta_{i}^{*}(s ; \tau) f_{3-i}^{*}\left(s+\lambda_{i}^{\prime}\right)\right]\right. \\
&+\varepsilon_{3-i}^{*}(s ; \tau) \cdot\left[\left(s+\lambda_{1}+\lambda_{2}+\lambda_{12}\right) \beta_{i}^{*}(s ; \tau)+k_{i}^{*}(s ; \tau) f_{i}^{*}\left(s+\lambda_{3-i}^{\prime}\right)\right] \\
&\left.+\varepsilon_{i}^{*}(s ; \tau)\left[s+\lambda_{1}+\lambda_{2}+\lambda_{12}-f_{3-i}^{*}\left(s+\lambda_{i}^{\prime}\right) k_{i}^{*}(s ; \tau)\right]\right\} .
\end{aligned}
$$

Hence we have

$$
\begin{aligned}
Q_{1(i)}^{* \infty}(s)= & D_{i}^{*}(s ; \tau)=\left\{\eta^{*}(s ; \tau)\left[f_{i}^{*}\left(s+\lambda_{3-i}^{\prime}\right)+\beta_{i}^{*}(s ; \tau) f_{3-i}^{*}\left(s+\lambda_{i}^{\prime}\right)\right]\right. \\
& +\varepsilon_{3-i}^{*}(s ; \tau)\left[\left(s+\lambda_{1}+\lambda_{2}+\lambda_{12}\right) \cdot \beta_{i}^{*}(s ; \tau)+k_{i}^{*}(s ; \tau) f_{i}^{*}\left(s+\lambda_{3-i}^{\prime}\right)\right] \\
& \left.+\varepsilon_{i}^{*}(s ; \tau)\left[\left(s+\lambda_{1}+\lambda_{2}+\lambda_{12}\right)-f_{3-i}^{*}\left(s+\lambda_{i}^{\prime}\right) k_{i}^{*}(s ; \tau)\right]\right\} \\
& /\left\{\left(s+\lambda_{1}+\lambda_{2}+\lambda_{12}\right)\left[1-\beta_{1}^{*}(s ; \tau) \beta_{2}^{*}(s ; \tau)\right]\right. \\
& \left.-\sum_{j=1}^{2} f_{j}^{*}\left(s+\lambda_{3-j}^{\prime}\right)\left[k_{3-j}^{*}(s ; \tau)+\beta_{3-j}^{*}(s ; \tau) k_{j}^{*}(s ; \tau)\right]\right\} .
\end{aligned}
$$

Noting that $k_{i}^{*}(s ; \tau)=\lambda_{3-i}+\lambda_{12} \alpha_{i} f_{i}^{\dagger}(s)$, and from (19) and (52) we have

$$
\begin{aligned}
Q_{2}^{* \infty}(s)= & {\left[\alpha_{1}^{*}(s)-\lambda_{2} f_{2}^{*}\left(s+\lambda_{1}^{\prime}\right)\right]^{-1}\left\{\sum_{j=1}^{2}\left[\lambda_{12} \alpha_{j} f_{j}^{\dagger}(s)+\lambda_{j} \beta_{j}^{*}(s ; \tau)\right] Q_{1(3-j)}^{* \infty}(s)\right.} \\
& \left.+\sum_{j=1}^{2} \lambda_{j} \varepsilon_{j}^{*}(s ; \tau)+\eta^{*}(s ; \tau)\right\} \\
= & {\left[\alpha_{1}^{*}(s)-\lambda_{2} f_{2}^{*}\left(s+\lambda_{1}^{\prime}\right)\right]^{-1}\left\{\left(s+\lambda_{1}+\lambda_{2}+\lambda_{12}\right)\left[1-\beta_{1}^{*}(s ; \tau) \beta_{2}^{*}(s ; \tau)\right]\right.} \\
& \left.-\sum_{j=1}^{2} f_{j}\left(s+\lambda_{3-j}^{\prime}\right)\left[k_{3-j}^{*}(s ; \tau)+\beta_{3-j}^{*}(s ; \tau) k_{j}^{*}(s ; \tau)\right]\right\}^{-1} \\
& \cdot\left\{\eta^{*}(s ; \tau)\left\{\left[\alpha_{1}^{*}(s)-\lambda_{2} f_{2}^{*}\left(s+\lambda_{1}^{\prime}\right)\right]\left[1-\beta_{1}^{*}(s ; \tau) \beta_{2}^{*}(s ; \tau)\right]\right\}\right\} \\
& +\sum_{j=1}^{2} \varepsilon_{j}^{*}(s ; \tau)\left\{\left[\alpha_{1}^{*}(s)-\lambda_{2} f_{2}^{*}\left(s+\lambda_{1}^{\prime}\right)\right]\left[k_{j}^{*}(s ; \tau) \beta_{3-i}^{*}(s ; \tau)+k_{3-j}^{*}(s ; \tau)\right]\right\}
\end{aligned}
$$

where

$$
\begin{aligned}
\sum_{j=1}^{2} \varepsilon_{j}^{*}(s ; \tau)\left[k_{j}^{*}(s ; \tau) \beta_{3-j}^{*}(s ; \tau)+k_{3-j}^{*}(s ; \tau)\right] \\
=\left[1-\beta_{1}^{*}(s ; \tau) \beta_{2}^{*}(s ; \tau)\right] \sum_{j=1}^{2}\left[\lambda_{3-j}+\lambda_{12} \alpha_{j} f_{j}^{\dagger}(s)\right] /\left(s+\lambda_{3-j}^{\prime}\right) \\
\quad-\sum_{j=1}^{2}\left\{\frac{\lambda_{3-j}^{\prime}}{s}\left\{k_{3-j}^{*}(s ; \tau)-k_{j}^{*}(s ; \tau)+\left[k_{j}^{*}(s ; \tau) \beta_{3-j}^{*}(s ; \tau)-k_{3-j}^{*}(s ; \tau) \beta_{j}^{*}(s ; \tau)\right]\right\}\right. \\
+\left[k_{j}^{*}(s ; \tau) \beta_{3-j}^{*}(s ; \tau)+k_{3-j}^{*}(s ; \tau)\right] \\
\\
\left.\quad \cdot\left[f_{j}^{*}\left(s+\lambda_{3-j}^{\prime}\right)+\lambda_{3-j}^{\prime}\left(\frac{e^{-s \tau}-1}{s}+F_{j}^{\dagger}(s)\right)\right]\right\} /\left(s+\lambda_{3-j}^{\prime}\right)
\end{aligned}
$$

Hence we have $\left(5^{* *}\right)$ from (53) and (54).

DERIVATION OF $\left(6^{* *}\right)$

After simple calculations we have

$$
\lambda_{12}+2 \lambda\left[1-f^{*}\left(\lambda^{\prime}\right)\right]=\frac{2 \lambda \lambda^{\prime}+\lambda_{12}\left(\lambda^{\prime}+\mu\right)}{\lambda^{\prime}+\mu},\left(2 \lambda+\lambda_{12}\right) \gamma^{*}(0, \tau)=\frac{\left(2 \lambda+\lambda_{12}\right)\left(1+\lambda^{\prime} \tau\right)+\mu\left(1+\lambda_{12} \tau\right)}{\lambda^{\prime}+\mu},
$$




$$
\begin{gathered}
\lambda_{12} F(\tau) f^{*}\left(\lambda^{\prime}\right)+\left(2 \lambda+\lambda_{12}\right) \beta^{*}(0 ; \tau)=\frac{\left[2 \lambda \lambda^{\prime}+\lambda_{12}\left(\lambda^{\prime}+\mu\right)\right]\left[1-e^{-\mu \tau}\right]}{\lambda^{\prime}+\mu}, \\
f^{*}\left(\lambda^{\prime}\right)\left[1+\lambda_{12}\left(\tau-\int_{0}^{\tau} F(t) d t\right)\right]+\left(2 \lambda+\lambda_{12}\right) \varepsilon^{*}(0 ; \tau) \\
=\frac{\mu\left[\mu+\lambda_{12}\left(1-e^{-\mu \tau}\right)\right]+\left(2 \lambda+\lambda_{12}\right)\left[\mu+\lambda^{\prime}\left(1-e^{\mu \tau}\right)\right]}{\mu\left(\lambda^{\prime}+\mu\right)}, \\
\left(2 \lambda+\lambda_{12}\right)\left[1-\beta^{*}(0 ; \tau)\right]-\left[2 \lambda+\lambda_{12} F(\tau)\right] f^{*}\left(\lambda^{\prime}\right)=\frac{\left[\mu \lambda_{12}+\lambda^{\prime}\left(2 \lambda+\lambda_{12}\right)\right] e^{-\mu \tau}}{\lambda^{\prime}+\mu}, \\
1+2 \lambda \varepsilon^{*}(0 ; \tau)+\lambda_{12}\left[\tau-\int_{0}^{\tau} F(t) d t\right]=\frac{\mu\left(2 \lambda+\lambda^{\prime}+\mu\right)+\left[2 \lambda \lambda^{\prime}+\lambda_{12}\left(\lambda^{\prime}+\mu\right)\right]\left(1-e^{-\mu \tau}\right)}{\mu\left(\lambda^{\prime}+\mu\right)},
\end{gathered}
$$

From (34), (35) and (55) we have (6**).

\section{References}

[1] J. Fukuta and M. Kodama, Mission reliability for a redundant repainable system with two dissimilar units, IEEE Trans. on Reliability, R-23 (1974), No. 4, 280-282.

[2] M. Kodama, J. Fukuta and S. Takamatsu, Mission reliability for a 1 -unit system with allowed down time, IEEE Trans. on Reliability, R-22 (1973), No. 5, 268-270. 